\title{
Migration of Viscoacoustic Data Using Acoustic Reverse Time Migration with Hybrid Deblurring Filters
}

\author{
Yuqing Chen*, Bowen Guo ${ }^{\dagger}$ and Gerard T. Schuster* \\ * Department of Earth Science and Engineering, \\ King Abdullah University of Science and Technology, \\ Thuwal, Saudi Arabia, 23955-6900. \\ † Microsoft(China) Co., Ltd, Beijing, China, \\ Email: yuqing.chen@kaust.edu.sa; Bowen.Guo@microsoft.com
}

(February 21, 2019)

Acoustic RTM with Hybrid Deblurring Filter

Running head: Acoustic RTM with Hybrid Deblurring Filter

\begin{abstract}
Viscoacoustic migration can significantly compensate for the amplitude loss and phase distortion in migration images computed from highly attenuated data. However, solving the viscoacoustic wave equation requires a significant amount of storage space and computation time, especially for least-squares migration methods. To mitigate this problem, we use acoustic reverse time migration (RTM) instead of viscoacoustic migration to migrate the viscoacoustic data, and then correct the amplitude and phase distortion by hybrid deblurring filters in the image domain. Numerical tests on synthetic and field data demonstrate that acoustic RTM combined with hybrid deblurring filters can compensate for the attenuation effects and produce images with high resolution and balanced amplitudes. This procedure requires less than $1 / 3$ of the storage space and is $O(N-1)$ times faster compared to the
\end{abstract}


viscoacoustic migration. Here, $\mathrm{N}$ represents the number of iterations used for least-square migration method. This method can be extended to $3 \mathrm{D}$ migration at even a greater cost saving. 


\section{INTRODUCTION}

Subsurface attenuation distorts both the amplitudes and phases of propagating waves. To correct for these distortions, several attenuation compensation methods were proposed to produce images with balanced amplitudes and accurately positioned reflectors (Dai and West, 1994; Blanch et al., 1995; Wang, 2007; Zhang et al., 2010; Valenciano et al., 2011; Suh et al., 2012; Fletcher et al., 2012; Dutta and Schuster, 2014; Dai et al., 2015; Sun et al., 2016; Chen et al., 2017).

For Q-reverse time migration (Q-RTM), Zhang et al. (2010), Suh et al. (2012), Zhu et al. (2014) and Zhu and Harris (2015) used a viscoacoustic wave equation which employs a fractional Laplacian operator to separately correct the phase and amplitude losses. Application of this equation to seismic data produces a higher resolution migration image with corrected amplitudes and phases by reversing the sign of the attenuation operator while keeping the sign of the dispersion operator unchanged. However, the disadvantage is that high-frequency noise will be amplified by the backward propagation of reflections which jeopardizes the image quality. To mitigate this problem, a low-pass filtering step is needed to stabilize the backward propagation by applying two-dimensional forward and inverse Fourier transforms to the wavefield at each time step. In addition, the spatial derivatives in the wave equation are calculated by the staggered-grid Fourier pseudo-spectral approach. Both of the techniques significantly increase the computation time.

Another method for compensating the attenuation effects is Q least-squares reverse time migration (Q-LSRTM) (Dutta and Schuster, 2014; Dai et al., 2015). This method computes the source-side wavefield based on a Q-propagator solved by a time-domain viscoacoustic wave equation based on the standard linear solid (SLS) model. However, the adjoint Q 
propagator used for backpropagating the data residual is also attenuative. This means a large number of iterations are required to improve the image quality, which makes the QLSRTM technique computationally expensive compared to standard RTM. Except for the data-domain method, seismic attenuation can be also compensated in the image-domain within the framework of depth-domain inversion (Cavalca et al., 2015; Fletcher et al., 2016).

To significantly reduce the computational cost of viscoacoustic migration, we propose applying a hybrid deblurring filter to the acoustic RTM image computed from attenuated data. Acoustic RTM is first used to migrate the lossy observed data, which results in a distorted image as acoustic RTM is unable to correct for the attenuation effects. Then the hybrid deblurring filters are used to correct the amplitude and phase distortions in the acoustic RTM image. These filters (Aoki and Schuster, 2009) can be calculated by the following procedure: (1) Construct a reference reflectivity model as a uniform distribution of point scatterers; (2) Generate the reference viscoacoustic data with the reference reflectivity model while the background velocity and Q models are the same as the ones used for migration; (3) Migrate the reference data by acoustic RTM to obtain a reference migration image; (4) Solve the hybrid deblurring filters by matching the reference migration image with the reflectivity model. If $\mathrm{N}$ iterations are required by Q-LSRTM, the hybrid deblurring is $O(N-1)$ times faster because no iterative model updates are required and this algorithm only computes solutions to the acoustic wave equation after the hybrid filter is computed.

This paper is divided into four sections. After the introduction, we present the theory for applying a hybrid deblurring filter to images computed by acoustic migration. Numerical tests on synthetic and field data are then used to demonstrate the advantages of the proposed method. The conclusions are presented in the last section. 


\section{THEORY}

For a homogeneous medium with velocity $v_{0}$ and a monochromatic point source at $\mathbf{x}_{s}=$ $\left(x_{s}, z_{s}\right)$ with angular frequency $\omega$, the viscoacoustic Green's function $G_{Q}\left(\mathbf{x}_{0}, \mathbf{x}_{s}\right)$ is given by

$$
G_{Q}\left(\mathbf{x}_{0}, \mathbf{x}_{s}\right)=\frac{\overbrace{\exp \left\{\frac{i \omega\left|\mathbf{x}_{s}-\mathbf{x}_{0}\right|}{v_{0} \xi}\right\}}^{\text {phase distortion }} \overbrace{\exp \left\{-\frac{\omega\left|\mathbf{x}_{s}-\mathbf{x}_{0}\right|}{2 Q v_{0} \xi}\right\}}^{\text {amplitude/frequency attenuation }}}{\left|\mathbf{x}_{s}-\mathbf{x}_{0}\right|},
$$

where

$$
\xi=\left[1+\frac{1}{\pi Q}\left(\ln \left(\frac{\omega}{\omega_{0}}\right)\right)\right]\left[1+\frac{1}{4 Q^{2}}\right]
$$

$Q$ is the quality factor which quantifies the attenuation in the subsurface, $\omega_{0}$ is the reference frequency (usually chosen to be the central frequency of the source wavelet), and $\mathbf{x}_{0}$ represents the subsurface location. The first exponential term is the phase distortion term and the second represents the amplitude/high-frequency loss. The scattered viscoacoustic data $\mathbf{d}_{Q}\left(\mathbf{x}_{g} \mid \mathbf{x}_{s}, \omega\right)$ under the Born approximation is a weighted summation of the Green's functions:

$$
d_{Q}\left(\mathbf{x}_{g} \mid \mathbf{x}_{s}, \omega\right)=\int_{V_{0}} w(\omega) G_{Q}\left(\mathbf{x}_{g} \mid \mathbf{x}_{0}, \omega\right) G_{Q}\left(\mathbf{x}_{0} \mid \mathbf{x}_{s}, \omega\right) m\left(\mathbf{x}_{0}\right) d V_{0}
$$

with the source at $\mathbf{x}_{s}$ and the receiver at $\mathbf{x}_{g} \cdot w(\omega)$ is the frequency-domain representation of the second-order time derivative of the source wavelet, and $m\left(\mathbf{x}_{0}\right)$ denotes the reflectivity distribution. Using matrix-vector notation, equation 3 can be re-written as

$$
\mathbf{d}_{Q}=\mathbf{L}_{Q} \mathbf{m}_{0}
$$


where $\mathbf{L}_{Q}$ is the viscoacoustic modeling operator whose kernel is given by $\left[w(\omega) G_{Q}\left(\mathbf{x}_{g} \mid \mathbf{x}_{0}, \omega\right) G_{Q}\left(\mathbf{x}_{0} \mid \mathbf{x}_{s}, \omega\right)\right]$ in equation 3. The migration image $\mathbf{m}_{m i g}$ is calculated by applying the adjoint operator $\mathbf{L}_{Q}^{T}$ to the data in equation 4 :

$$
\mathbf{m}_{m i g}=\mathbf{L}_{Q}^{T} \mathbf{d}_{Q}=\mathbf{L}_{Q}^{T} \mathbf{L}_{Q} \mathbf{m}_{0}
$$

where $\mathbf{L}_{Q}^{T}$ is the viscoacoustic adjoint operator which compensates for the amplitude loss and phase distortion. Equation 5 is denoted as the equation for Q-reverse time migration (Q-RTM).

\section{Q-RTM with Constant Q Wave Equation}

Zhang et al. (2010), Suh et al. (2012), (Zhu et al., 2014) and Zhu and Harris (2015) proposed a constant $\mathrm{Q}$ time-domain viscoacoustic wave equation for Q-RTM with a fractional Laplacian operator:

$$
\left(\eta \mathbf{L}+\tau \mathbf{H} \frac{d}{d t}-v^{-2} \frac{\partial^{2}}{\partial t^{2}}\right) P(t)=f(t)
$$

where $\mathbf{L}=\left(-\nabla^{2}\right)^{\gamma+1}$ represents the wave dispersion effect associated with rock attenuation and $\mathbf{H}=\left(-\nabla^{2}\right)^{\gamma+\frac{1}{2}}$ accounts for frequency-dependent amplitude attenuation. The absorption and dispersion coefficients are given by $\eta=-v^{2 \gamma} \omega_{\gamma}^{-2 \gamma} \cos \pi \gamma$ and $\tau=-v^{2 \gamma-1} \omega_{r}^{-2 \gamma} \sin \pi \gamma$, where $\left.\gamma=\frac{1}{\pi} \tan ^{-1}\left(\frac{1}{Q}\right)\right)$. By simply reversing the sign of the amplitude loss term and keeping the sign of the dispersion term unchanged, the energy of the receiver-side backpropagating wavefields will be amplified, and both amplitude loss and phase distortion are corrected. However, this amplification will also boost high-frequency noise and compro- 
mise the quality of the migration image. Therefore a low-pass filter is needed to stabilize the back-propagation operation which requires two-dimensional forward and inverse Fourier transforms at each time step. Moreover, the staggered-grid Fourier pseudo-spectral method is used to calculate the spatial derivative. Both these steps significantly increase the computational cost of the Q-RTM method.

\section{Q-LSRTM with SLS-model-based Wave Equation}

Q-LSRTM is another way to compensate for the attenuation effects by using the viscoacoustic wave equation derived from the SLS model:

$$
\begin{aligned}
\frac{\partial r_{l}}{\partial t} & =-\frac{r_{l}}{\tau_{\sigma l}}+\frac{K}{\tau_{\sigma l}}\left(1-\frac{\tau_{\epsilon l}}{\tau_{\sigma l}}\right) v_{x} \\
-\frac{\partial P}{\partial t} & =K\left[1-\frac{1}{L} \sum_{l=1}^{L}\left(1-\frac{\tau_{\epsilon l}}{\tau_{\sigma} l}\right)\right] v_{x}+\frac{1}{L} \sum_{l=1}^{L} r_{l}+S \\
-\frac{1}{\rho} \frac{\partial p}{\partial x} & =\frac{\partial v_{x}}{\partial t}
\end{aligned}
$$

where $K$ denotes the bulk modulus of the medium, $S$ indicates the source wavelet, and $\tau_{\epsilon l}$ and $\tau_{\sigma l}$ are the strain and stress relaxation times, respectively. $r_{l}$ denotes the memory variables and $l=1, \ldots, L$ is the number of viscoacoustic mechanisms in the wave equation. Unfortunately, the computation time and storage requirements will increase with the number of attenuation mechanisms. Another problem is that the adjoint propagator used to backpropagate the data residual is also attenuative, so that the receiver-side backpropagated wavefields will be further attenuated. As a result, a large number of least-squares iterations are required to obtain the desired image quality. Empirically, the Q-LSRTM is at 
least three times more computationally expensive compared with standard acoustic RTM in a non-attenuative medium.

\section{Acoustic RTM with Hybrid Deblurring Filters}

Acoustic RTM is a computational friendly method because it is easy to implement and numerically stable. Migrating viscoacoustic data using the acoustic migration can be formulated as

$$
\tilde{\mathbf{m}}_{m i g}=\mathbf{L}^{T} \mathbf{d}_{Q}=\mathbf{L}^{T} \mathbf{L}_{Q} \mathbf{m}_{0}
$$

where $\mathbf{L}^{T}$ is the acoustic migration operator and $\tilde{\mathbf{m}}_{m i g}$ is the migration image. The tilde symbol indicates that the migration image $\tilde{\mathbf{m}}_{m i g}$ is incorrect as the acoustic migration operator is not able to compensate for the phase distortion and amplitude loss in the migration image. Equation 8 can be re-written as

$$
\tilde{m}_{m i g}(\mathbf{x})=\sum_{\mathbf{x}_{0}} \Gamma\left(\mathbf{x} \mid \mathbf{x}_{\mathbf{0}}\right) m\left(\mathbf{x}_{0}\right)
$$

where, $\Gamma_{m i g}\left(\mathbf{x} \mid \mathbf{x}_{0}\right)$ can be explicitly derived in a homogeneous medium with constant velocity $\mathbf{v}_{0}$ as

$$
\begin{aligned}
\Gamma_{m i g}\left(\mathbf{x} \mid \mathbf{x}_{0}, \omega\right) & =\int_{\omega} d \omega \sum_{s} \sum_{r} G^{*}\left(\mathbf{x}_{g} \mid \mathbf{x}\right) G^{*}\left(\mathbf{x} \mid \mathbf{x}_{s}\right) G_{Q}\left(\mathbf{x}_{g} \mid \mathbf{x}_{0}\right) G_{Q}\left(\mathbf{x}_{0} \mid \mathbf{x}_{s}\right) \\
& =\int_{\omega} d \omega \sum_{s} \sum_{r} \frac{\exp \left\{i \frac{\omega}{v_{0}}\left(\frac{r r}{\xi}-r r_{0}\right)\right\} \exp \left\{-\frac{\omega}{2 Q v_{0} \xi}(r r)\right\}}{\left|\mathbf{x}_{s}-\mathbf{x}\right| \cdot\left|\mathbf{x}_{r}-\mathbf{x}\right| \cdot\left|\mathbf{x}_{s}-\mathbf{x}_{0}\right| \cdot\left|\mathbf{x}_{r}-\mathbf{x}_{0}\right|}
\end{aligned}
$$

where $r r=\left|\mathbf{x}_{s}-\mathbf{x}\right|+\left|\mathbf{x}_{r}-\mathbf{x}\right|, r r_{0}=\left|\mathbf{x}_{s}-\mathbf{x}_{0}\right|+\left|\mathbf{x}_{r}-\mathbf{x}_{0}\right|$ and the symbol $*$ indicates complex conjugation. Equation 10 suggests that if acoustic migration is used to migrate viscoacoustic data, the reflection energy will be focused at the wrong location where $\frac{r r}{\xi}-r r_{0}=0$. 
Furthermore, the image is blurred by $\mathbf{L}^{T} \mathbf{L}_{Q}$. To compensate for the attenuation effects and insure that the imaged reflectors are at the right location in the $\tilde{\mathbf{m}}_{m i g}$ image, the inverse of the Hessian operator $\left(\mathbf{L}^{T} \mathbf{L}_{Q}\right)^{-1}$ needs be applied to the migration image, so that

$$
\begin{aligned}
\mathbf{m}_{\text {deblur }} & =\left(\mathbf{L}^{T} \mathbf{L}_{Q}\right)^{-1} \tilde{\mathbf{m}}_{\text {mig }} \\
& =\left(\mathbf{L}^{T} \mathbf{L}_{Q}\right)^{-1}\left(\mathbf{L}^{T} \mathbf{L}_{Q}\right) \mathbf{m}_{0} \\
& =\mathbf{m}_{0}
\end{aligned}
$$

However, it is computationally prohibitive to compute the direct inverse of $\mathbf{L}^{T} \mathbf{L}_{Q}$ for practical seismic imaging problems. In addition, $\mathbf{L}^{T} \mathbf{L}_{Q}$ is often ill-conditioned so that its inverse is prone to numerical errors.

We now propose a hybrid deblurring filter to efficiently approximate the Hessian inverse operator $\left(\mathbf{L}^{T} \mathbf{L}_{Q}\right)^{-1}$. To estimate the deblurring filter, we first define a uniform distribution of point scatterers as the reference reflectivity model. The viscoacoustic synthetic data $\mathbf{d}_{Q}$ are generated using this reference reflectivity model with the background velocity and $\mathrm{Q}$ models:

$$
\mathbf{d}_{r e f_{-} Q}=\mathbf{L}_{Q} \mathbf{m}_{r e f}
$$

The data $\mathbf{d}_{r e f_{-} Q}$ are then migrated by acoustic RTM to obtain a reference migration image

$$
\tilde{\mathbf{m}}_{\text {mig_ref }}=\mathbf{L}^{T} \mathbf{d}_{r e f_{-} Q} \text {. }
$$

The hybrid deblurring filters $\mathbf{F}_{i}$ for different subdomains of the RTM image are then esti- 
mated by locally matching the reference migration image $\tilde{\mathbf{m}}_{m i g_{r e f}}$ with the true reference reflectivity model $\mathbf{m}_{r e f}$ as

$$
\left[\mathbf{m}_{\text {ref }}\right]_{i}=[\mathbf{F}]_{i} \otimes\left[\tilde{\mathbf{m}}_{\text {mig_ref }}\right]_{i}
$$

where $\otimes$ denotes spatial convolution and $i$ indicates the $i$ th local window. Here, $[\mathbf{F}]_{i}$, $\left[\mathbf{m}_{r e f}\right]_{i}$, and $\left[\tilde{\mathbf{m}}_{\text {mig_ref }}\right]_{i}$ denote the deblurring filter, the reference reflectivity model, and the reference migration image within the $i$ th local window, respectively. To numerically estimate the deblurring filter, equation 14 needs to be transformed into matrix multiplication form

$$
\left[\mathbf{M}_{r e f_{-} m i g}\right]_{i}[\mathbf{f}]_{i}=\left[\mathbf{m}_{r e f}\right]_{i} .
$$

This requires flattening the two-dimensional deblurring filter $[\mathbf{F}]_{i}$ into the vector format $[\mathbf{f}]_{i}$ and transforming the migration image $\left[\mathbf{m}_{m i g_{-} r e f}\right]_{i}$ into a convolution matrix $\left[\mathbf{M}_{r e f \_m i g}\right]_{i}$. This transformation is shown in Figure 1, where the yellow migration "butterfly" indicates the migration response $\left[\tilde{\mathbf{m}}_{\text {mig_ref }}\right]_{i}$ of a point scatterer in the $i$ th local window. The red square with the dimensions $w_{i n} \times w_{i n}$ represents the local window which should effectively cover the migration butterfly. The blue square with the dimensions $f_{x} \times f_{z}$ represent the hybrid deblurring filter. In principle, the size of the filter should entirely cover the migration butterfly. However, in that case, a large deblurring filter is needed which dramatically increases the computational cost. To reduce this cost we use a deblurring filter that is no larger than several dominant wavelengths in the migrated data, so this deblurring filter mitigates high-and intermediate-wavenumber artifacts, but not the low-wavenumber one. The hybrid deblurring filter can be estimated by solving equation 15 by the least-squares 
method: (Aoki and Schuster, 2009; Dai and Schuster, 2009; Fletcher et al., 2016)

$$
[\mathbf{f}]_{i}=\left(\left[\mathbf{M}_{r e f \_m i g}\right]_{i}^{T}\left[\mathbf{M}_{r e f \_m i g}\right]_{i}\right)^{-1}\left[\mathbf{M}_{r e f \_m i g}\right]_{i}^{T}\left[\mathbf{m}_{r e f}\right]_{i},
$$

and the estimated filters can then be applied to the real migration image $\tilde{\mathbf{m}}_{\text {mig }}$ to correct for the attenuation and blurring effects:

$$
\left[\mathbf{m}_{\text {deblur }}\right]_{i} \approx[\mathbf{F}]_{i} \otimes \tilde{\mathbf{m}}_{m i g}
$$

\section{WORKFLOW}

The workflow for migration of viscoacoustic data using acoustic RTM with a hybrid deblurring filters consists of the following steps:

1. Compute $\tilde{\mathbf{m}}_{m i g}$ by migrating the viscoacoustic observed data using acoustic RTM.

2. Build a reference reflectivity model $\mathbf{m}_{r e f}$ with point scatterers evenly distributed in the model. The reference viscoacoustic data $\mathbf{d}_{r e f_{-} Q}$ are generated by the viscoacoustic Born modeling based on this reference reflectivity model and the background velocity and Q models.

3. Compute the reference migration image $\tilde{\mathbf{m}}_{\text {mig_ref }}$ by migrating the reference data using acoustic RTM.

4. The hybrid deblurring filters $\mathbf{F}_{i}$ for different subsections of the RTM image are estimated by locally matching the reference migration image $\left[\tilde{\mathbf{m}}_{m i g \_r e f}\right]_{i}$ with the reference reflectivity model $\left[\mathbf{m}_{r e f}\right]_{i}$. 
5. Apply the calculated filters $\mathbf{F}_{i}$ to the migration image $\tilde{\mathbf{m}}_{m i g}$ to correct for the attenuation distortions.

\section{NUMERICAL RESULTS}

The effectiveness of migrating viscoacoustic data by the combination of acoustic RTM and the hybrid deblurring filter is now demonstrated with synthetic and field data examples. The data are migrated using acoustic RTM, Q-RTM, Q-LSRTM and acoustic RTM with hybrid deblurring filters. The migration results are compared with the same colorbar scale.

\section{Point-Scatterer Model}

A point-scatterer model is used to test the hybrid filter's effectiveness in correcting for image distortion. The size of the model is 201 by 201 grid points with a single point scatterer located in the middle of a homogeneous model with $v=2500 \mathrm{~m} / \mathrm{s}$ and $Q=25$; and the seismic data are computed using equation 4 with a $15-\mathrm{Hz}$ Ricker wavelet. Figure 2a shows the migration image using acoustic RTM to migrate the lossy data. Compared with the actual scatterer location, which is indicated by the red star, the migration response of the

point scatterer is mis-located. After applying the hybrid deblurring filters, these errors have been successfully corrected in Figure $2 \mathrm{~b}$.

\section{Sensitivity of Deblurred Images with Respect to Errors in the Attenuation Model}

To check the sensitivity of the hybrid deblurring filters to errors in the estimated Q model, numerical tests are carried out based on the previous scatterer model for different back- 
ground $\mathrm{Q}$ values.

Figure 3a shows the migration image migrated by acoustic RTM and the red star indicates the correct point-scatterer location. The attenuation distortions in Figure 3a are corrected in Figure 3b by applying the hybrid deblurring filters to the acoustic RTM image. In this case, these filters are estimated based on the correct background Q model. However, the hybrid deblurring filters become less effective as the errors of the estimated Q model increase.

To illustrate this point, Figure 4 shows the performance of the deblurring filter with respect to different errors in the estimated $\mathrm{Q}$ model by comparing the deblurred image to the reference image. The reference image is obtained by applying the acoustic LSRTM to the acoustic data. As the errors in the $\mathrm{Q}$ model increase, the similarity between the deblurred image and the reference image decreases quadratically, where the similarity is quantified as the correlation between the deblurred image and the reference image. We can see that when the error is larger than 40 percent, the hybrid deblurring filter becomes less effective in correcting the attenuation distortions.

Therefore, a reliable Q model is required for the hybrid compensation method. For datadomain methods, Quan and Harris (1997) used the centroid-frequency shifts between the predicted and the observed traces and smeared the shifts along raypaths to update the $\mathrm{Q}$ model. For full waveform inversion (FWI)-like algorithm, Bai et al. (2014) and Cheng* et al. (2015) used the waveform difference to invert for the Q model. Dutta and Schuster (2016) developed a skeletonized wave-equation $\mathrm{Q}$ inversion method that finds the $\mathrm{Q}$ model that minimizes the differences of the peak frequencies between the observed and the predicted transmission arrivals. This skeletonized method is less prone to the cycle skipping compared 
to the waveform difference misfit function. For image-domain algorithms, Shen* et al. (2014) invert for the $\mathrm{Q}$ model by minimizing the spectral ratio difference between a $\mathrm{Q}$-image and a target image, which is attenuation-free. The above Q inversion methods provide a varity of options for estimating the Q model that is needed for the hybrid compensation method.

\section{Effectiveness and Limitations of the Hybrid Compensation}

We now test the deblurring filter on migration images generated from different $\mathrm{Q}$ models. Figures 5a, 5c, 5e and 5g show the acoustic migration results with the observed datasets generated from the homogeneous attenuation models with the $1 / \mathrm{Q}=0.01,0.04,0.07$ and 0.1 , respectively. The amplitudes and phase distortions in the acoustic migration image become increasingly severe as $1 / \mathrm{Q}$ increases. We then apply the hybrid deblurring filters to these images to correct the attenuation distortions and the results are shown in Figures $5 \mathrm{~b}, 5 \mathrm{~d}, 5 \mathrm{f}$ and $5 \mathrm{~h}$. In all four cases, the amplitude and phase distortions in these images are well recovered. The blue curve in Figure 6 represents the similarity between the hybrid compensated images and the reference image. Here, the reference image is obtained by applying acoustic LSRTM to acoustic data. We can see that as the attenuation increases from $1 / \mathrm{Q}=0.05$ to $1 / \mathrm{Q}=0.1$, the performance of the hybrid deblurring filter decreased by $22 \%$, which is acceptable compared to a migration image without compensation. The reason for the performance degradation is that as the attenuation increase, the spatial resolution and the amplitude of the migration Green's function decrease, so that solving for the hybrid deblurring filters becomes more ill-conditioned.

A key assumption with the deblurring filter is that the migration Green's function is spatially invariant in the filter window. This assumption is violated in a geophysical medium 
with a strong spatial heterogeneity, so appropriate remedies must be used. One such remedy is to overlap the adjacent filter windows and to make the windows smaller. However, these windows cannot be too small otherwise the filters will not be able to compensate for intermediate-wavenumber distortions. In this paper, the reference model is a set of uniformly distributed point scatterers. There are other options. For example, a reference model which follows the geology of the model may have the potential benefit over the uniform one (Aoki and Schuster, 2009).

\section{Marmousi II Model}

Acoustic RTM with hybrid deblurring filters is now tested on viscoacoustic data generated for the Marmousi II model. Figure 7 shows the true velocity and 1/Q models. A Ricker wavelet with a peak frequency of $15 \mathrm{~Hz}$ is used as the source wavelet and a fixed-spread acquisition geometry is employed where 150 sources are evenly distributed on the surface at a source interval of $50 \mathrm{~m}$. The data are recorded by 800 receivers for each shot, where the receivers are uniformly distributed every $10 \mathrm{~m}$ on the surface.

Figure 8a shows the conventional acoustic RTM image computed from viscoacoustic data. This image fails to recover the correct amplitudes of the deeper reflectors, and some reflectors are located at the wrong position. The Q-LSRTM image, shown in Figure 8b, shows an improvement in the deeper layers when compared to the acoustic RTM result. However, the Q-LSRTM image has a lower resolution for those reflectors below the Q anomaly compared to the benchmark migration image shown in Figure 8d. More iterations are needed for Q-LSRTM to get a more balanced image with higher resolution. Here, the benchmark image is the result of acoustic LSRTM applied to acoustic data generated 
from the same velocity model in Figure 7a. To mitigate the amplitude-loss and phase-shift problems in the acoustic RTM image, we apply the hybrid deblurring filters to the image in Figure 8a. The result is shown in Figure 8c, which has better resolution with more balanced amplitudes, and the reflectors are at the correct locations.

Magnified views of these images are compared in Figures 9 and 10, where the red arrows point to the areas with noticeable improvements. The correct location of the reflectors are indicated by the blue dashed lines in Figures 9 and 10, where the reflectors in Figures 9a and 10a are at the wrong places compared to the other images. This is due to the fact that the acoustic migration operator cannot correct the phase-shift problem in the lossy data. In contrast, the hybrid deblurring filter successfully corrects for phase and amplitude distortions as shown in Figures 9c and 10c.

\section{Friendswood Crosswell Field Data}

We now test our method on the Friendswood crosswell field data set. Two 305-m-deep cased wells separated by $183 \mathrm{~m}$ were used as the source and receiver wells. Downhole explosive charges were fired at intervals of $3 \mathrm{~m}$ from $9 \mathrm{~m}$ to $305 \mathrm{~m}$ in the source well, and the receiver well had 96 receivers placed at depths ranging from $3 \mathrm{~m}$ to $293 \mathrm{~m}$. For these data, the migration velocity and $\mathrm{Q}$ models are shown in Figures $11 \mathrm{a}$ and $11 \mathrm{~b}$, respectively. The migration velocity model is estimated by early-arrival waveform inversion and the migration Q model is estimated by wave-equation Q tomography (Dutta and Schuster, 2016).

The comparison between the acoustic RTM and Q-LSRTM images are shown in Figures $12 \mathrm{a}$ and $12 \mathrm{~b}$, respectively. Similar to the synthetic examples, the amplitudes are more balanced in the Q-LSRTM image in Figure 12b than those in the acoustic RTM image in 
Figure 12a. At the depths of $25 \mathrm{~m}$ and $48 \mathrm{~m}$, two strong and continuous reflectors are shown in the Q-LSRTM image, which are barely visible in the RTM image. By applying the hybrid deblurring filter to Figure 12a, these reflectors are recovered in the deblurred image. Moreover, Figure 12c also suggests that the amplitudes in the deblurred image also become more balanced and the phase problem is largely mitigated.

On the other hand, there are also some artifacts in the deblurred image. At the depth of $250 \mathrm{~m}$, there are three continuous reflectors in the Q-LSRTM image in Figure 12b, and yet these reflectors can hardly be seen at the right part of the deblurred image in Figure 12c. The possible reason is that the estimated $Q$ model is not accurate enough in this area.

Figure 13 depicts the magnified views of the black box in Figure 12, where the arrows point to the areas with noticeable improvements. Some missing reflectors in the RTM image are well recovered by the hybrid deblurring filter in the deblurred image shown in Figure 13c. Figure 13a shows a deviation of the migrated reflector from the correct location (marked by the red line). This shift is corrected in Figure 13c by the hybrid deblurring filter. The comparison of the vertical profiles in Figure 12 at $\mathrm{x}=68 \mathrm{~m}$ are shown in Figure 14, where the acoustic migration result (blue line) is weakly correlated with the Q-LSRTM image (red line) in Figure 14a. However, after applying the hybrid deblurring filter to the acoustic migration image, the deblurred image (black line) correlates well with the Q-LSRTM image (red line) in Figure 14b.

\section{CONCLUSIONS}

An acoustic hybrid RTM method is presented that uses hybrid deblurring filters to compensate for the amplitude losses and phase shifts due to strong attenuation in the subsurface. 
Numerical tests on synthetic and field data show that the proposed method can significantly mitigate the problem of amplitude loss and phase shift in acoustic RTM images when migrating lossy data. This technique also increases spatial resolution in the migration image and can reduce the computational costs and storage requirements by a factor of $O(N-1)$ times compared to iterative Q-LSRTM. Here, $\mathrm{N}$ is the number of iterations.

The limitations of this method are that the hybrid deblurring filters are more suitable for correcting high- and intermediate-wavenumber distortion errors in a migration image caused by inadequate compensation for attenuation distortion. Compensating for the lowwavenumber errors with a larger deblurring filter will significantly increase the computational costs. For strong distortions, such as using acoustic migration on strongly anisotropic data, the hybrid deblurring filters are not able to correct for these effects. The performance of the hybrid deblurring filter relies on the accuracy of the estimated Q model, otherwise, will degrade in performance with decreased accuracy in the Q model.

\section{ACKNOWLEDGMENTS}

The research reported in this paper was supported by the King Abdullah University of Science and Technology (KAUST) in Thuwal, Saudi Arabia. We are grateful to the sponsors of the Center for Subsurface Imaging and Modeling (CSIM) Consortium for their financial support. For computer time, this research used the resources of the Supercomputing Laboratory at KAUST. We thank them for providing the computational resources required for carrying out this work. We also thank Exxon for the Friendswood crosswell data. 


\section{REFERENCES}

Aoki, N. and G. T. Schuster, 2009, Fast least-squares migration with a deblurring filter: Geophysics, 74(6), WCA83-WCA93.

Bai, J., D. Yingst, R. Bloor, and J. Leveille, 2014, Viscoacoustic waveform inversion of velocity structures in the time domainviscoacoustic waveform inversion: Geophysics, 79(3), R103-R119.

Blanch, J. O., J. O. Robertsson, and W. W. Symes, 1995, Modeling of a constant Q: Methodology and algorithm for an efficient and optimally inexpensive viscoelastic technique: Geophysics, 60(1), 176-184.

Cavalca, M., R. Fletcher, and X. Du, 2015, Q-compensation through depth domain inversion: 77th Conference and Exhibition, EAGE, Extended Abstracts, http://dx.doi.org/10.3997/2214-4609.201413250.

Chen, Y., G. Dutta, W. Dai, and G. T. Schuster, 2017, Q-least-squares reverse time migration with viscoacoustic deblurring filters: Geophysics, 82(6), S425-S438.

Cheng*, X., K. Jiao, D. Sun, and D. Vigh, 2015, A new approach of visco-acoustic waveform inversion in the time domain: 85th Annual International Meeting, SEG, Expanded Abstracts, 1183-1187.

Dai, N. and G. F. West, 1994, Inverse Q migration: 64th Annual International Meeting, SEG, Expanded Abstracts, 1418-1421.

Dai, W. and G. T. Schuster, 2009, Least-squares migration of simultaneous sources data with a deblurring filter: 79th Annual International Meeting, SEG, Expanded Abstracts, $2990-2994$.

Dai, W., Z. Xu, and R. Coates, 2015, Least-squares reverse-time migration for visco-acoustic media: 85th Annual International Meeting, SEG, Expanded Abstracts, 3387-3391. 
Dutta, G. and G. T. Schuster, 2014, Attenuation compensation for least-squares reverse time migration using the viscoacoustic-wave equation: Geophysics, 79(6), S251-S262.

—, 2016, Wave-equation Q tomography: Geophysics, 81(6), R471-R484.

Fletcher, R., D. Nichols, and M. Cavalca, 2012, Wavepath-consistent effective Q estimation for Q-compensated reverse-time migration: 74th Conference and Exhibition, EAGE, Extended Abstracts, 2214-4069-1487.

Fletcher, R. P., D. Nichols, R. Bloor, and R. T. Coates, 2016, Least-squares migrationdata domain versus image domain using point spread functions: The Leading Edge, 35, 157162.

Quan, Y. and J. M. Harris, 1997, Seismic attenuation tomography using the frequency shift method: Geophysics, 62(3), 895-905.

Shen*, Y., B. Biondi, R. Clapp, and D. Nichols, 2014, Wave-equation migration Q analysis WEMQA: 84th Annual International Meeting, SEG, Expanded Abstracts, 3757-3762.

Suh, S., K. Yoon, J. Cai, and B. Wang, 2012, Compensating visco-acoustic effects in anisotropic resverse-time migration: 82th Annual International Meeting, SEG, Expanded Abstracts, 1-5.

Sun, J., S. Fomel, T. Zhu, and J. Hu, 2016, Q-compensated least-squares reverse time migration using low-rank one-step wave extrapolation: Geophysics, 81(4), S271-S279.

Valenciano, A., N. Chemingui, D. Whitmore, and S. B. Dahl, 2011, Wave equation migration with attenuation and anisotropy compensation: 81th Annual International Meeting, SEG, Expanded Abstracts, 232-236.

Wang, Y., 2007, Inverse-Q filtered migration: Geophysics, 73(1), S1-S6.

Zhang, Y., P. Zhang, and H. Zhang, 2010, Compensating for visco-acoustic effects in reversetime migration: 80th Annual International Meeting, SEG, Expanded Abstracts, 3160- 
3164.

Zhu, T. and J. M. Harris, 2015, Improved seismic image by Q-compensated reverse time migration: Application to crosswell field data, West Texas: Geophysics, 80(2), B61-B67.

Zhu, T., J. M. Harris, and B. Biondi, 2014, Q-compensated reverse-time migration: Geophysics, 79(3), S77-S87. 


\section{LIST OF FIGURES}

1 Migration response of a point scatterer in the $i$ th local window and the convolution matrix associated with the image.

2 Comparison between images from (a) acoustic RTM and (b) acoustic RTM with a hybrid deblurring filter.

3 Comparison between (a) acoustic RTM image, deblurred images with (b) $\mathrm{Q}=25$, (c) $\mathrm{Q}=100$ and (d) $\mathrm{Q}=10000$, respectively.

4 Sensitivity of deblurred image with respect to the errors in the attenuation model.

5 Acoustic migration results with the observed datasets generated by the homogeneous attenuation model with $1 / \mathrm{Q}=$ (a) 0.01, (c) 0.04, (e) 0.07 and (g) 0.1 , respectively. The corresponding deblurred results are shown in (b), (d), (f) and (h).

6 Effectiveness of the hybrid deblurring and the attenuation factor $1 / \mathrm{Q}$.

$7 \quad$ The Marmousi model: (a) true velocity model and (b) true Q model.

8 Comparison between images computed from the viscoacoustic data by (a) acoustic RTM, (b) Q-LSRTM, (c) acoustic RTM with hybrid deblurring filters and (d) acoustic LSRTM for lossless acoustic data, which is used as the benchmark image. For these leastsquares images, 20 iterations are carried out in all cases.

$9 \quad$ Magnified views of the red boxes in Figure 8. The red arrows point to the areas with noticeable improvements and the blue dashed line indicates the true locations of the reflectors.

10 Magnified views of the black boxes in Figures 8. The red arrows point to the areas with noticeable improvements and the blue dashed line indicates the true locations of the reflectors.

11 (a) The estimated migration velocity and (b) Q models for the Friendswood cross- 
well data.

12 Comparison between the images from (a) acoustic RTM, (b) Q-LSRTM and (c) acoustic RTM with hybrid deblurring filters.

13 Magnified views of the black boxes in Figure 12.

14 Comparison of the vertical profiles in Figure 12 at $\mathrm{x}=68 \mathrm{~m}$. 
Transformation of Migration Image into Convolution Matrix
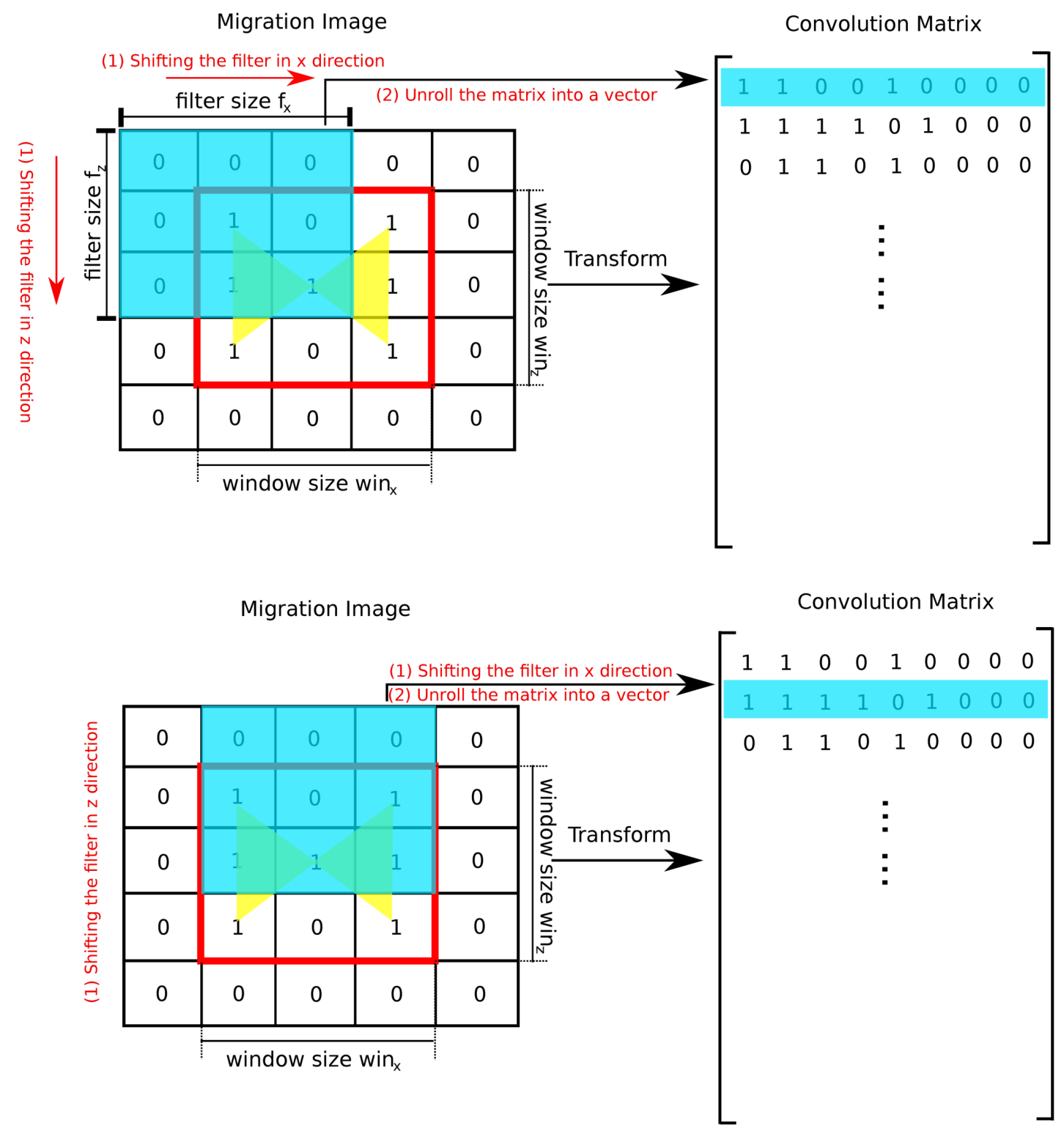

Figure 1: Migration response of a point scatterer in the $i$ th local window and the convolution matrix associated with the image. 
(a) Acoustic RTM

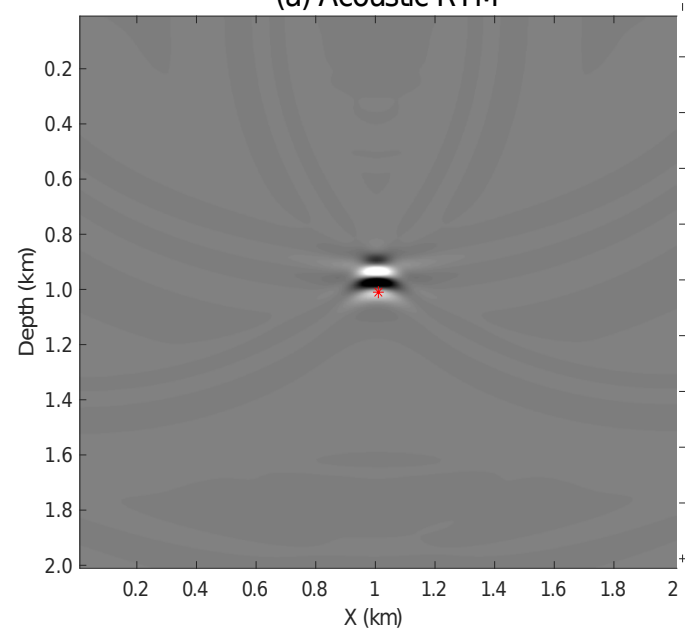

(b) Acoustic RTM with Hybrid Debluming Filter

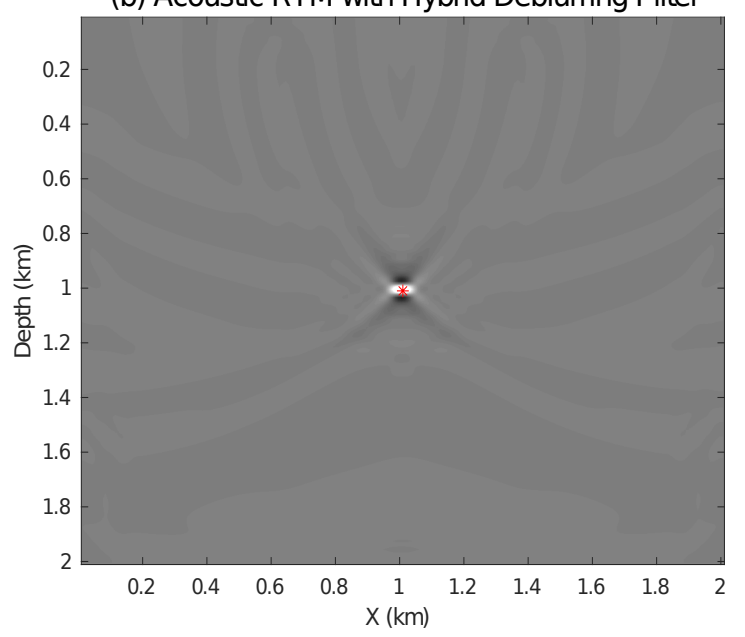

Figure 2: Comparison between images from (a) acoustic RTM and (b) acoustic RTM with a hybrid deblurring filter. 
(a) Acoustic RTM

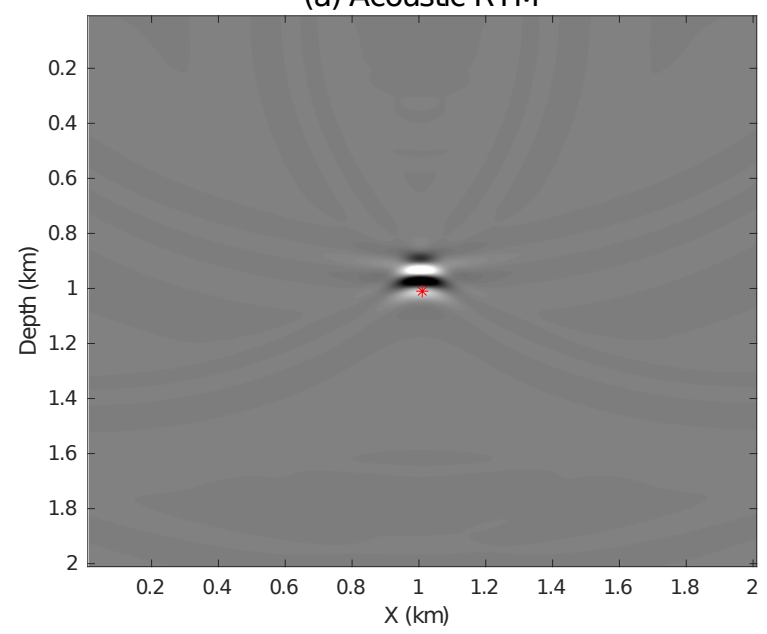

(c) RTM with Hybrid Debluming Filter $(\mathrm{Q}=100)$

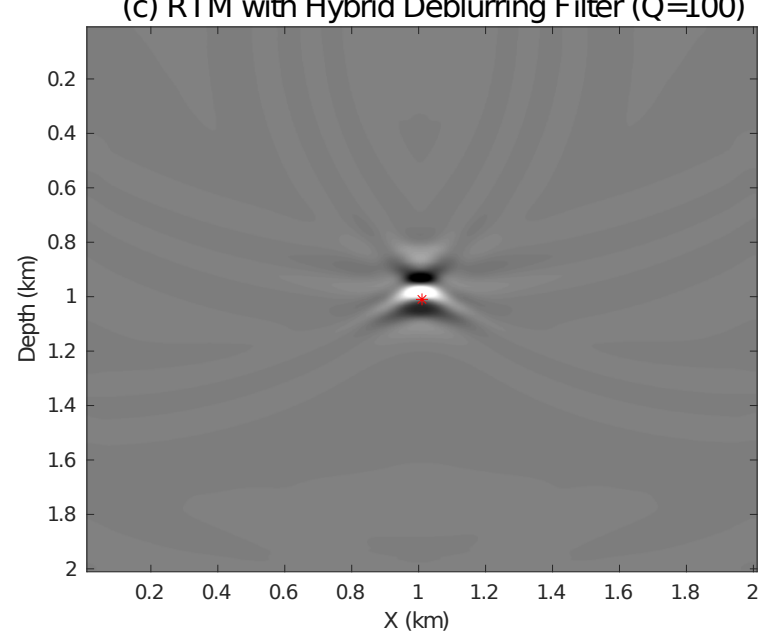

(b) RTM with Hybrid Debluming Filter ( $Q=25)$

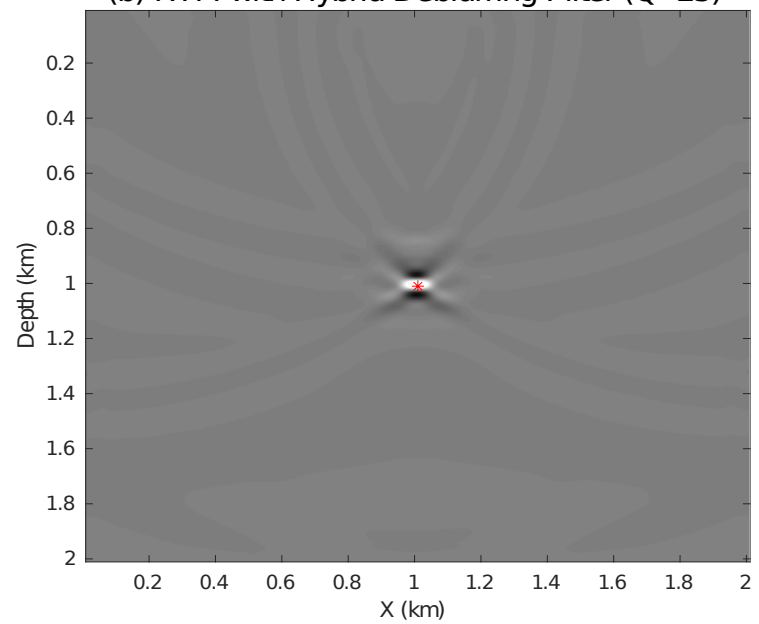

(d) RTM with Hybrid Deblurring Filter $(\mathrm{Q}=10000)$

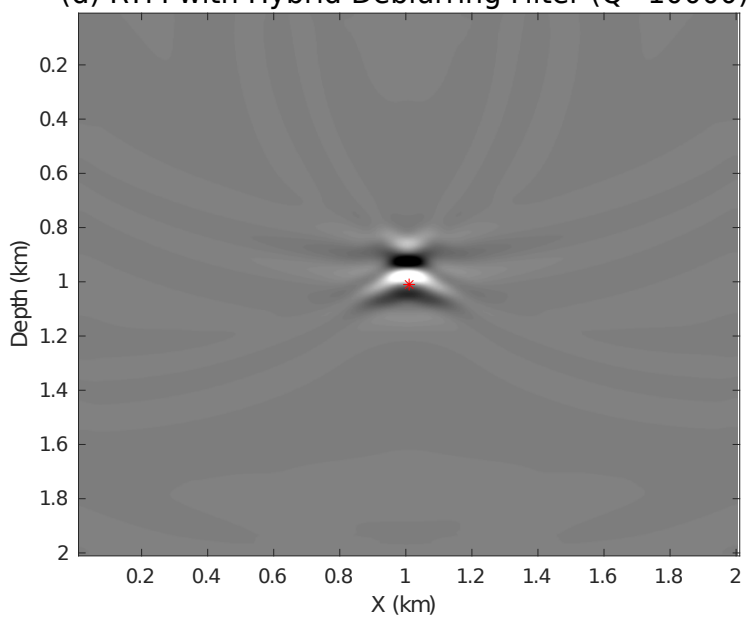

Figure 3: Comparison between (a) acoustic RTM image, deblurred images with (b) $\mathrm{Q}=25$, (c) $\mathrm{Q}=100$ and (d) $\mathrm{Q}=10000$, respectively. 


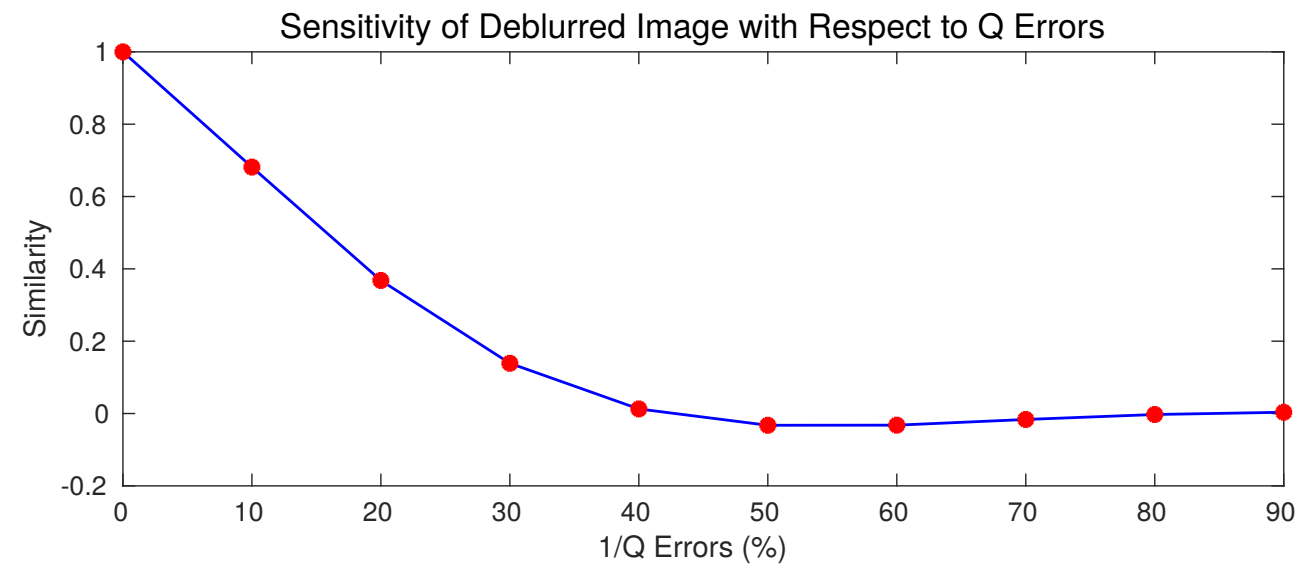

Figure 4: Sensitivity of deblurred image with respect to the errors in the attenuation model. 
(a) Acoustic Image with $1 / \mathrm{Q}=0.01$

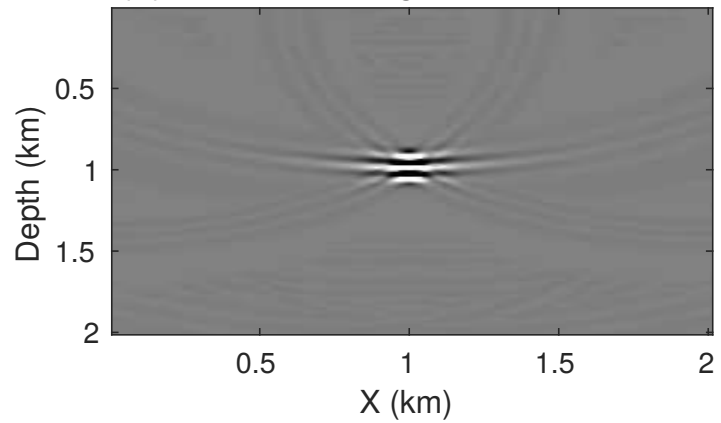

(c) Acoustic Image with $1 / Q=0.04$

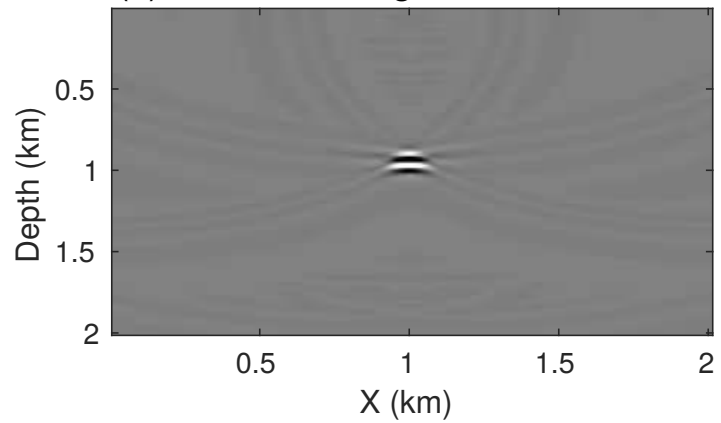

(e) Acoustic Image with $1 / \mathrm{Q}=0.07$

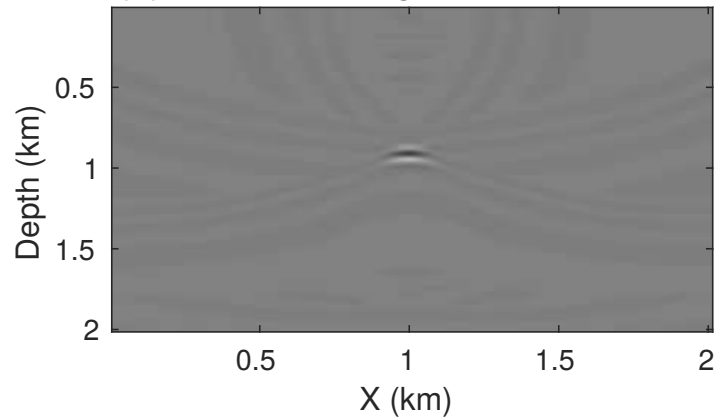

(g) Acoustic Image with $1 / Q=0.1$

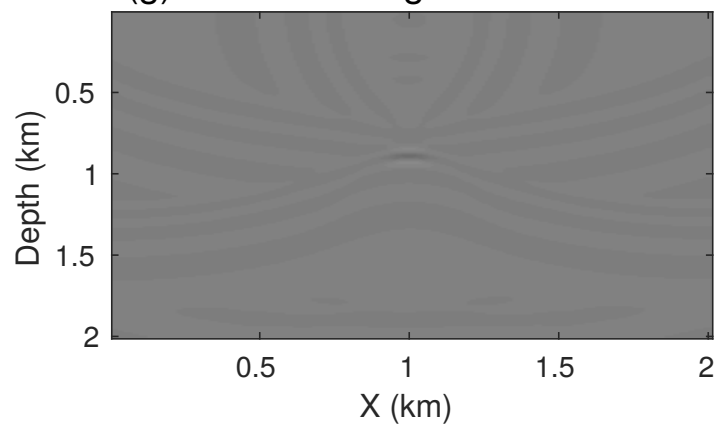

(b) Deblurred Image with $1 / \mathrm{Q}=0.01$

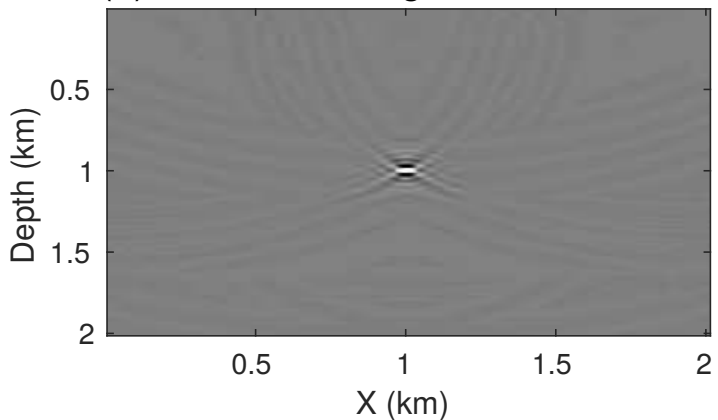

(d) Deblurred Image with $1 / \mathrm{Q}=0.04$

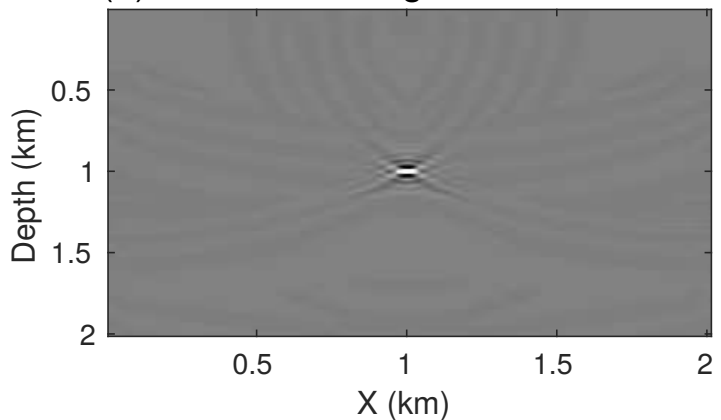

(f) Deblurred Image with $1 / \mathrm{Q}=0.07$

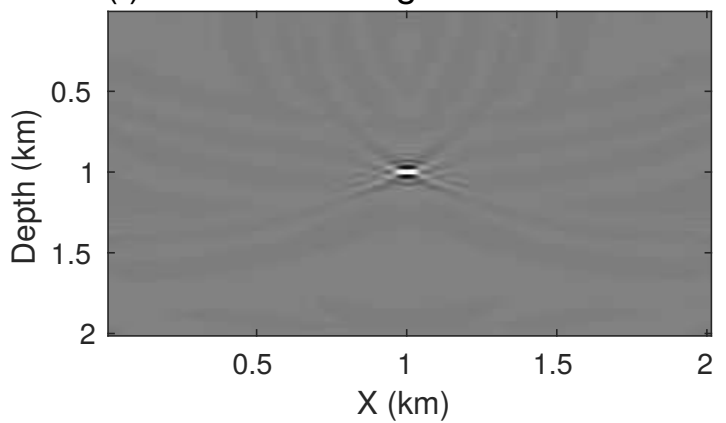

(h) Deblurred Image with $1 / \mathrm{Q}=0.1$

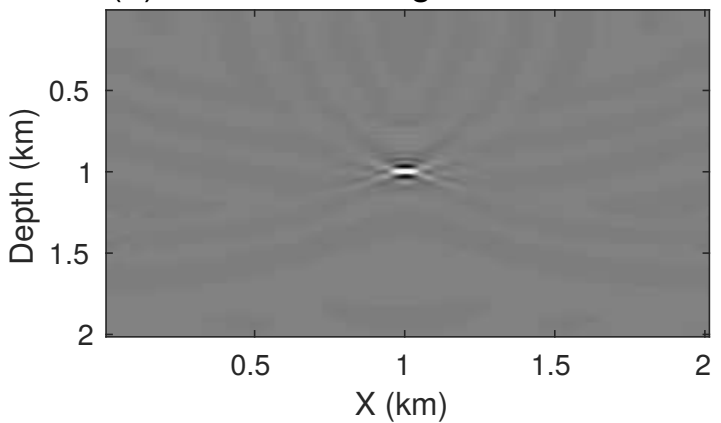

Figure 5: Acoustic migration results with the observed datasets generated by the homogeneous attenuation model with $1 / \mathrm{Q}=$ (a) 0.01 , (c) 0.04 , (e) 0.07 and (g) 0.1 , respectively. The corresponding deblurred results are shown in (b), (d), (f) and (h). 


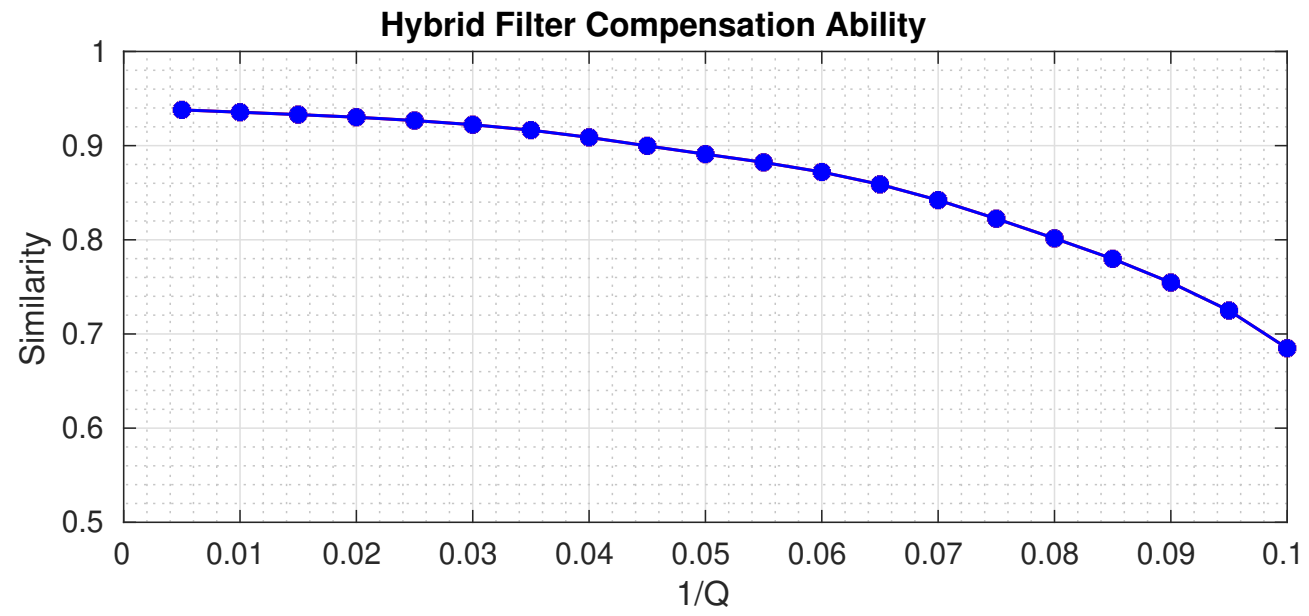

Figure 6: Effectiveness of the hybrid deblurring and the attenuation factor 1/Q. 

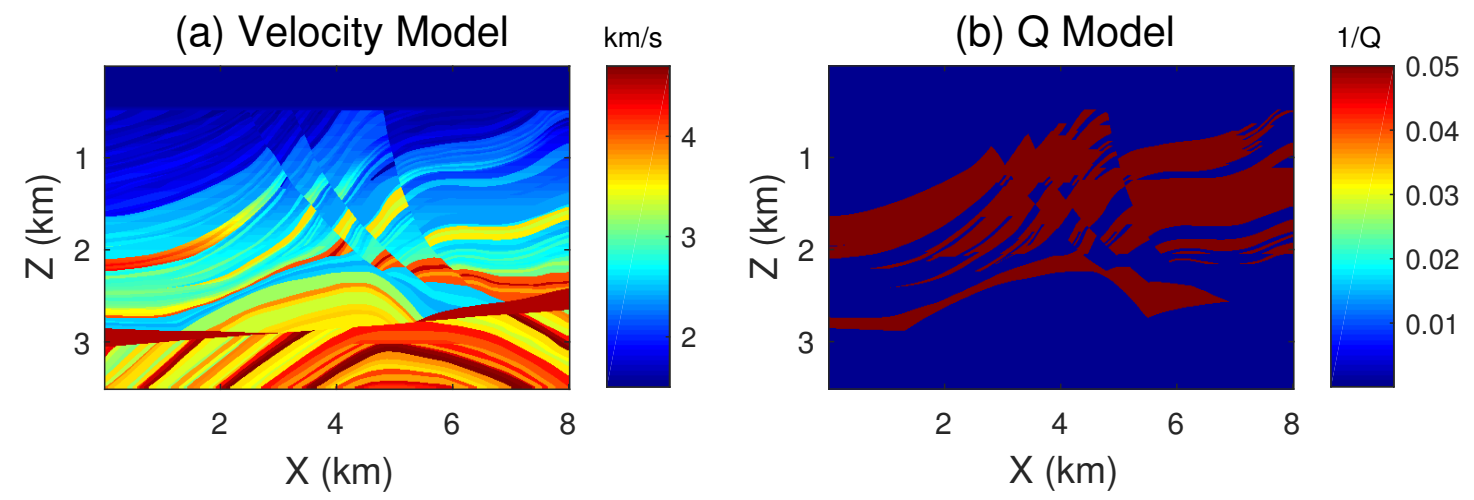

Figure 7: The Marmousi model: (a) true velocity model and (b) true Q model. 
(a) RTM

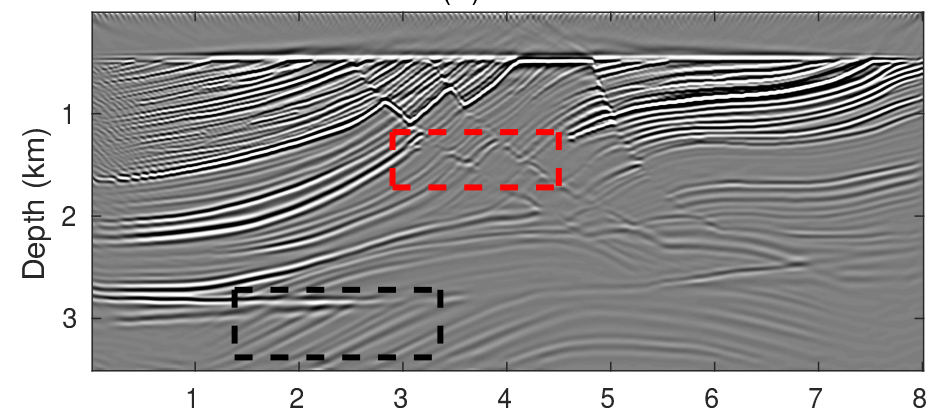

(b) Q-LSRTM

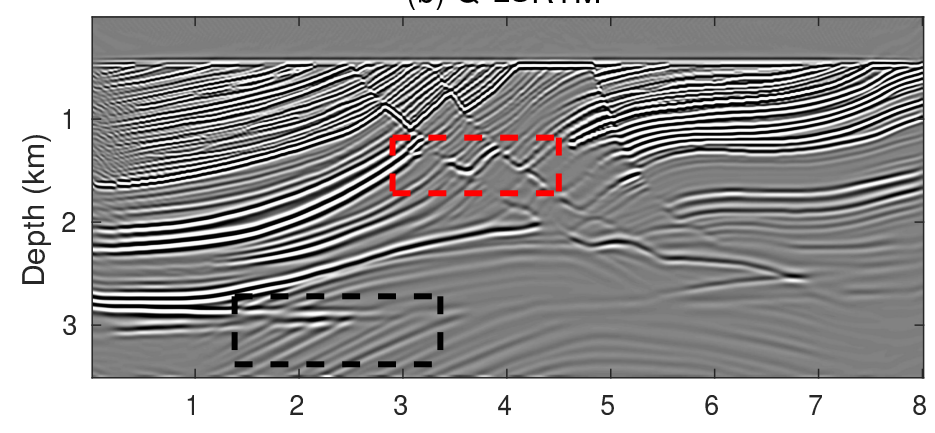

(c) RTM with Hybrid Deblurring Filters

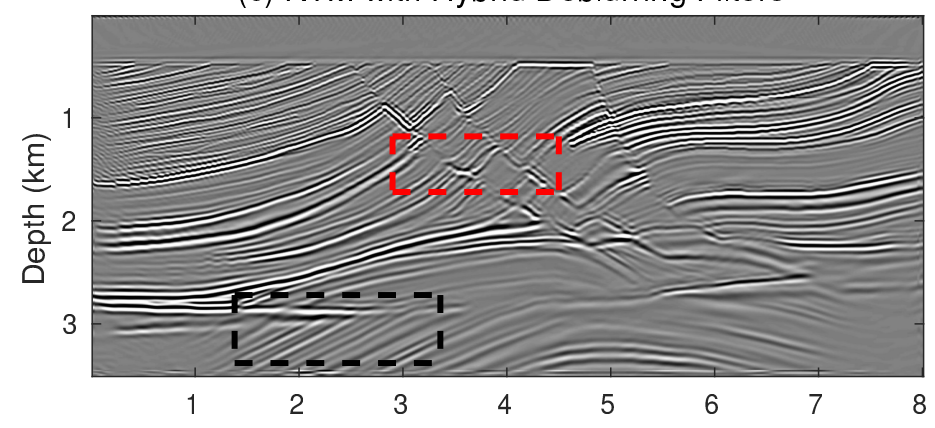

(d) Benchmark LSRTM

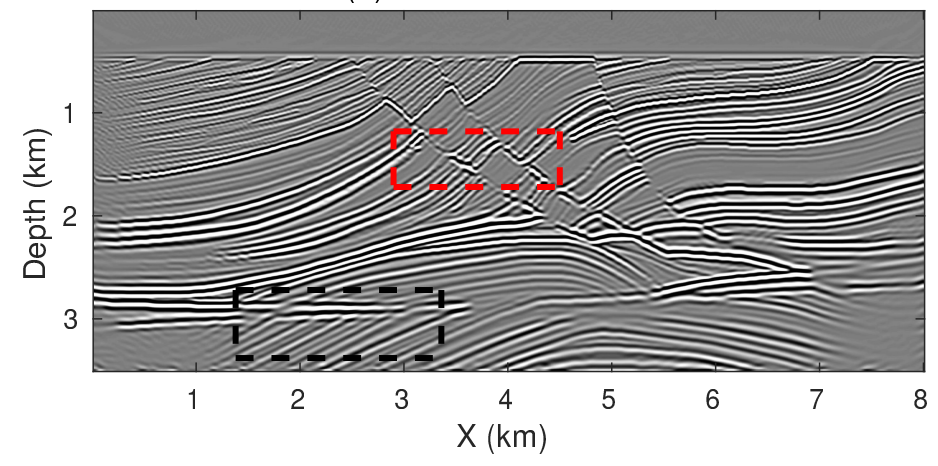

Figure 8: Comparison between images computed from the viscoacoustic data by (a) acoustic RTM, (b) Q-LSRTM, (c) acoustic RTM with hybrid deblurring filters and (d) acoustic LSRTM for lossless acoustic data, which is used as the benchmark image. For these leastsquares images, 20 iterations are carried out in all cases. 
(a) RTM

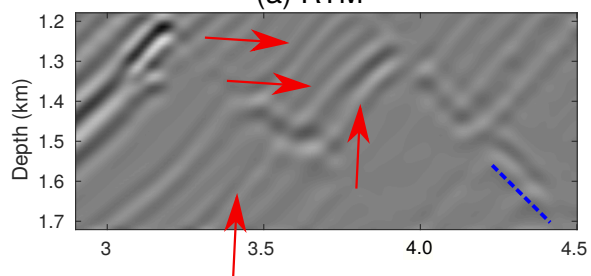

(c) RTM with Hybrid Deblurring Filters

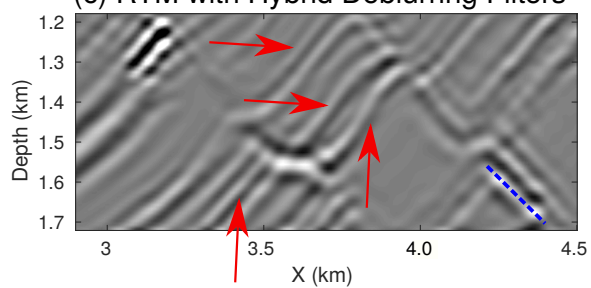

(b) Q-LSRTM

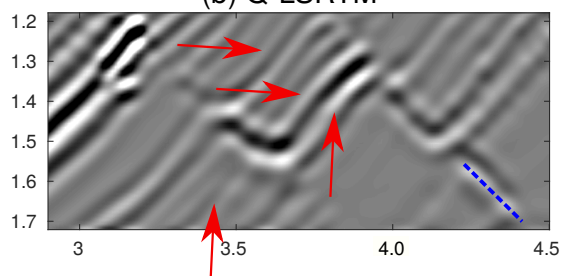

(d) Benchmark LSRTM

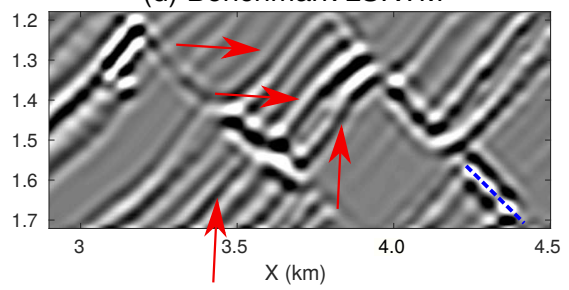

Figure 9: Magnified views of the red boxes in Figure 8. The red arrows point to the areas with noticeable improvements and the blue dashed line indicates the true locations of the reflectors. 
(a) RTM

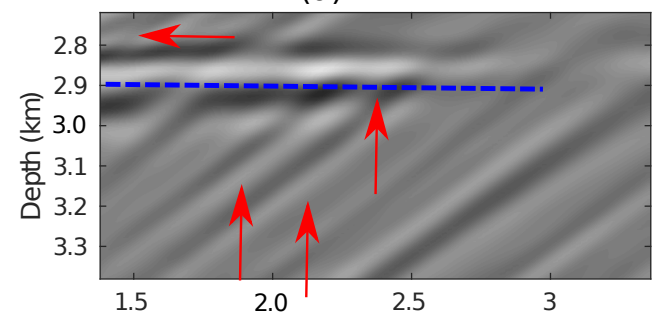

(c) RTM with Hybrid Deblurring Filters

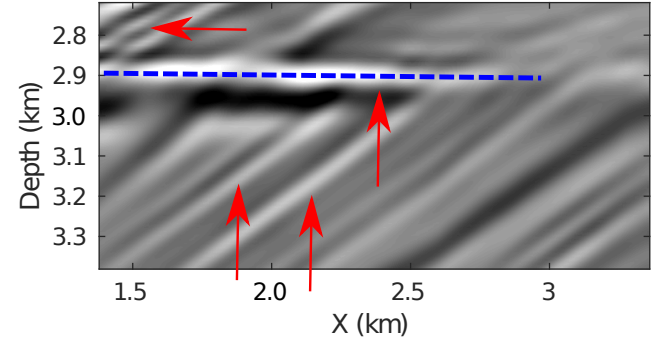

(b) Q-LSRTM

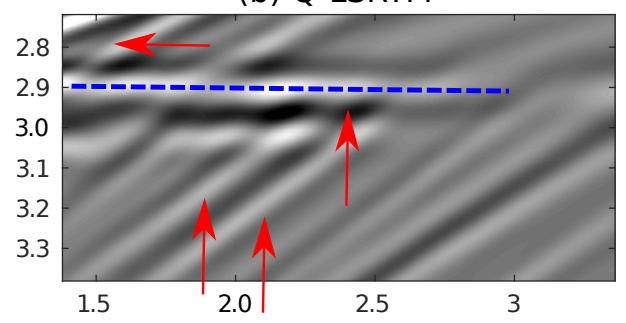

(d) Benchmark LSRTM

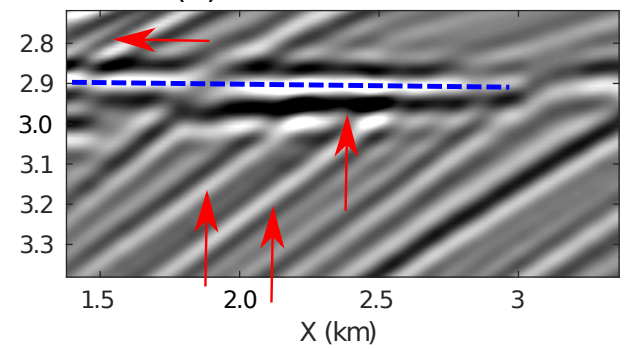

Figure 10: Magnified views of the black boxes in Figures 8. The red arrows point to the areas with noticeable improvements and the blue dashed line indicates the true locations of the reflectors. 


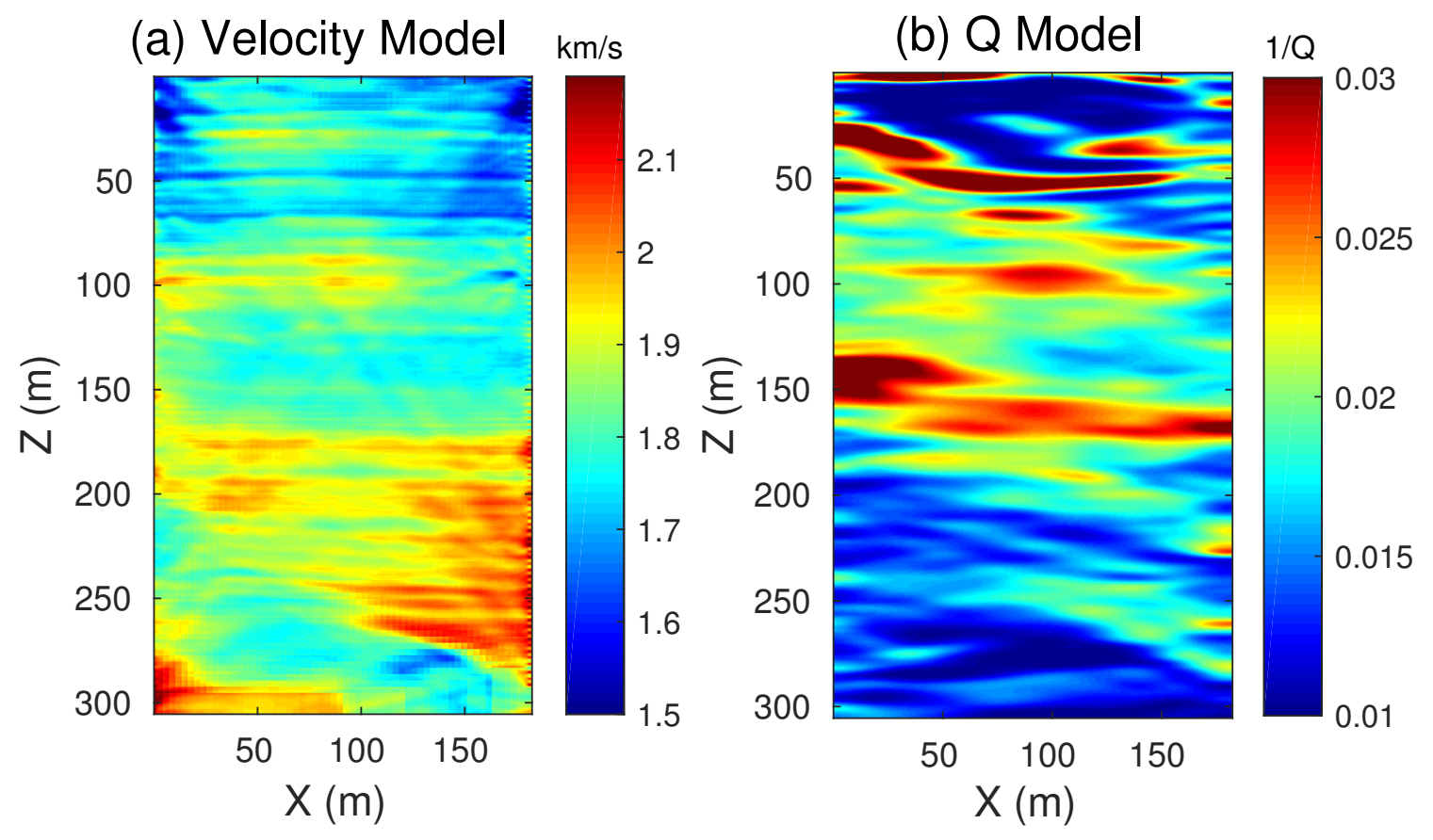

Figure 11: (a) The estimated migration velocity and (b) Q models for the Friendswood crosswell data. 

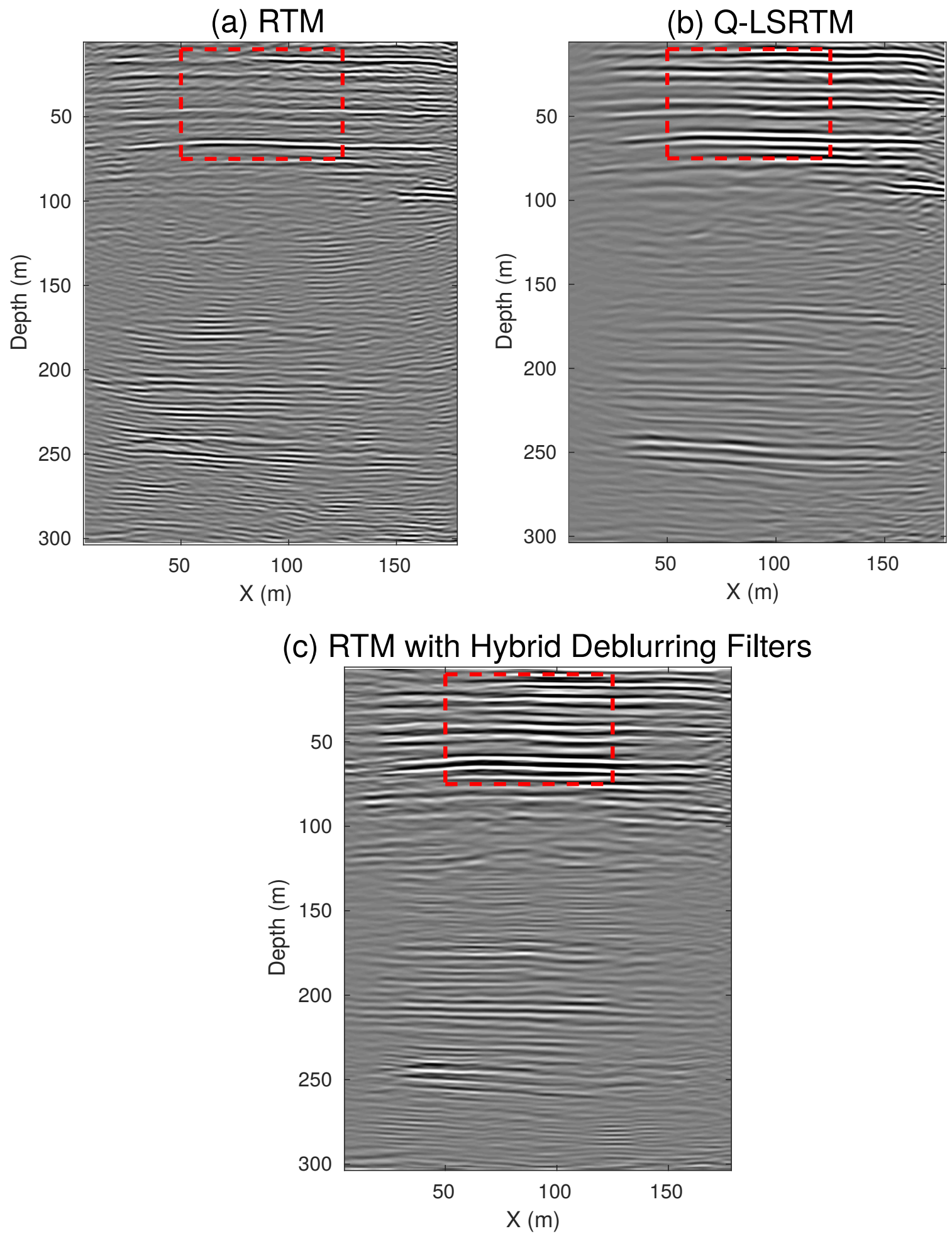

Figure 12: Comparison between the images from (a) acoustic RTM, (b) Q-LSRTM and (c) acoustic RTM with hybrid deblurring filters. 


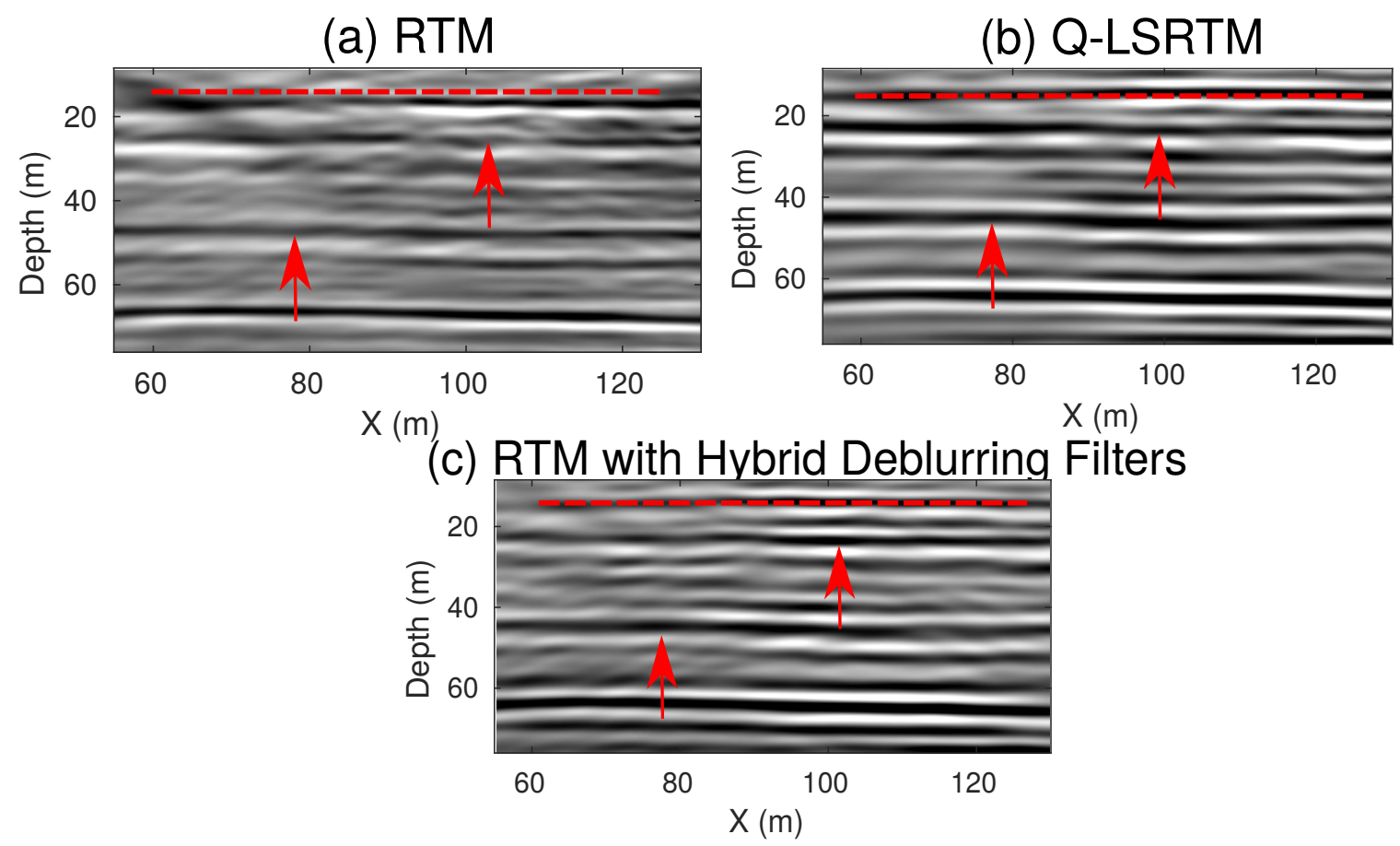

Figure 13: Magnified views of the black boxes in Figure 12. 
(a) Q-LSRTM vs Acoustic RTM

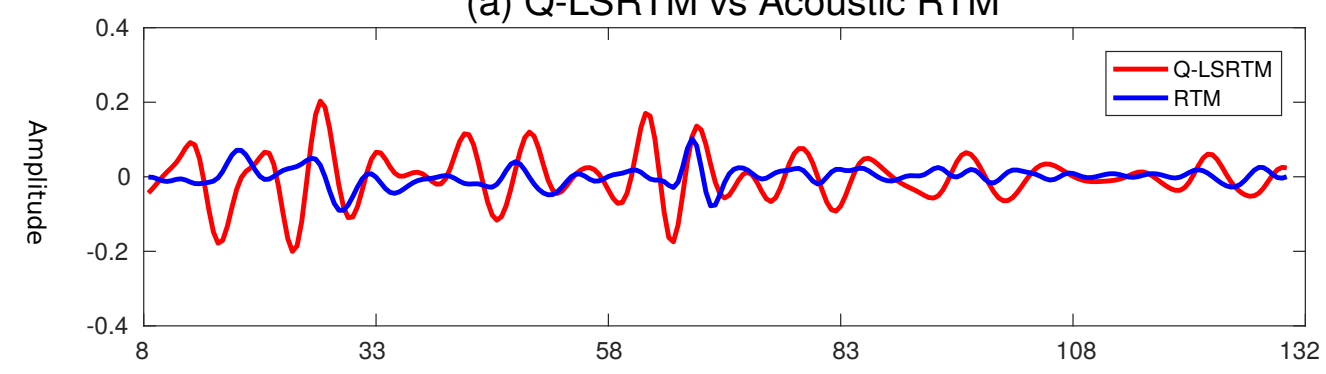

(b) Q-LSRTM vs Acoustic RTM with Hybrid Deblurring Flilters

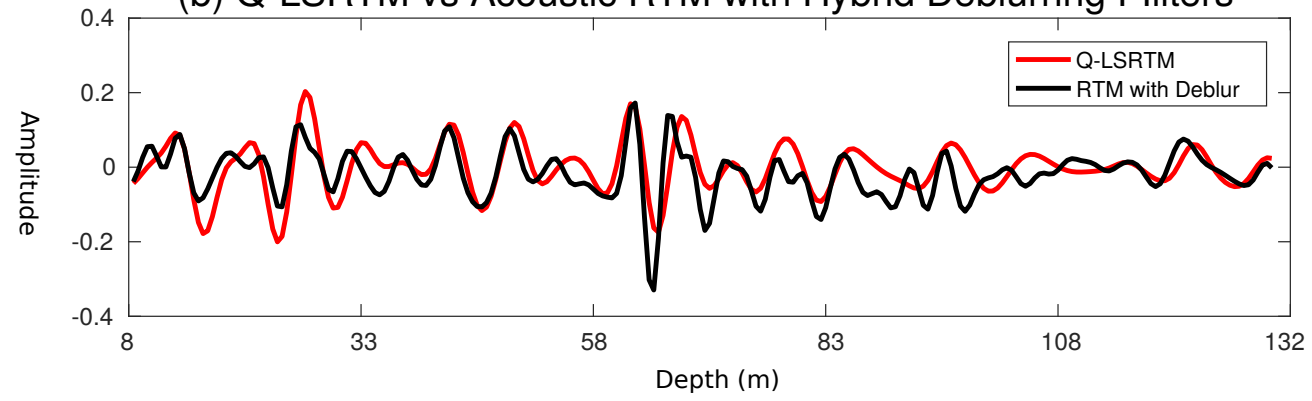

Figure 14: Comparison of the vertical profiles in Figure 12 at $\mathrm{x}=68 \mathrm{~m}$. 